\title{
El cemento portland en la consistencia del hormigón fresco. Finura de molido óptima
}

M. SABESINSKY FELPERIN:

\author{
R E S U M E N
}

En el presente trabajo, se investiga en micro-hormigones frescos preparados con esqueletos granulares compactos y agregados normales, la influencia de la finura de molido del cemento portland en la consistencia del cuerpo compuesto fresco, de distintas relaciones agua/cemento.

La relación agua/cemento de la pasta conglomerante y la finura de molido del cemento portland componente de la misma, gobiernan las propiedades del hormigón fresco.

Para una determinada consistencia puede establecerse "una finura de molido óptima del cemento portland", la cual permite reducir a un mínimo los requerimientos de agua de amasado.

Estimando la finura de molido por mediciones de superficie específica puede establecerse que, para consistencias del hormigón fresco dentro del rango plástico, la superficie específica óptima corresponde a valores entre 2.900 y $3.060 \mathrm{~cm}^{2} / \mathrm{g}$ (BLAINE).

\section{OBJETO DEL PRESENTE TRABAJO}

Este trabajo, es parte de un amplio Plan de Investigación tendente a establecer relaciones de semejanza entre hormigones preparados con esqueletos granulares de distintos tamaños máximos (micro, normal y macroesqueleto), e investigar las propiedades mecánicas y físico-químicas a través del micro-hormigón (mortero tipo), empleando técnicas e instrumental más adecuados a la búsqueda de determinados parámetros micro-estructurales.

En este trabajo, se considera la influencia de la finura de molido del cemento portland en la consistencia del cuerpo compuesto fresco, para diustintas relaciones agua/cemento de la pasta conglomerante.

* Profesor Titular (Investigador) - Jefe del Laboratorio de Tecnología de Materiales en el Instituto de Mecánica Aplicada y Estructuras (IMAE) - Facultad de Ciencias Exactas e Ingeniería-U.N. de Rosario (Argentina). 


\section{INTRODUCCION}

En el presente trabajo, se investiga en micro-hormigones frescos la influencia de la finura de molido del cemento portland, estimada por mediciones de superficie específica $\left(\mathrm{cm}^{2} / \mathrm{g}\right.$ BLAINE).

En trabajos previos (1), (2), (3), (4), (5) y (6) se ha puesto en evidencia la infiuencia que tiene la superficie especifica del cemento portland en las propiedades del hormigón fresco, principalmente en su consistencia. Rebinder (7) señala que los materiales finamente pulverizados adquieren nuevas propiedades, y que en el caso del cemento portland, con la conveniente pulverización fina, se permitirá dirigir los procesos de fraguado y de endurecimiento.

De acuerdo con Jaspers (8), las propiedades de la pasta de cemento portland, tanto fresca como endurecida, resultan ser función de la composición mineralógica del cemento y de su superficie específica. La resistencia mecánica, la estabilidad de volumen, la resistencia química (durabilidad) y el tiempo de fraguado resultan ser función de la superficie específica del cemento portland. En relación al fraguado, tanto en el comienzo como en el final del proceso, el tiempo correspondiente decrece a medida que se incrementa la superficie específica del cemento. Asimismo, el calor generado durante el proceso mencionado resulta ser función lineal del logarítmo de la superficie específica del cemento portland.

En relación al cemento portland y al agua de amasado, se ha concluido en base a las investigaciones llevadas a cabo en trabajos previos (4), (9), (10), (11) y (12), que ambos deben ser considerados en forma conjunta, en lugar de estimar al primero como a un material granular más, entre los componentes del hormigón a elaborar. De acuerdo con Richartz (13), y con las observaciones conductimétricas llevadas a cabo para el examen del proceso de fraguado de las pastas y mortero frescos de cemento portland en distintas condiciones higrotérmicas (2), (3), (4) y (6), la influencia de la pasta de cemento se hace sentir desde el momento en que sus componentes (cemento y agua de amasado) se incorporan al conjunto granular compuesto.

En consecuencia, considerando a la pasta de cemento, por una parte, y a los agregados gruesos y finos, por la otra, se puede estimar al hormigón de cemento portland como a un cuerpo compuesto de dos fases. Los agregados granulares, compuestos de partículas de diferentes tamaños y formas (esqueleto granular), constituyen la fase interna, discontinua o carga, en un medio fase externa o matriz, que puede llegar a ser continua (pasta conglomerante). En el caso de empleo de aditivos incorporadores intencionales de aire, las micro-burbujas presentes compensan las deficiencias en la granulometría del agregado fino y actúan como tal. Este sistema de micro-burbujas incoalescentes se considera parte componente del esqueleto granular.

La viscosidad de la pasta conglomerante resulta ser función de su relación "agua/cemento". En esta relación, se tiene el factor de mayor influencia en las propiedades mecánicas y físico-químicas del hormigón obtenido. Si la compactación del hormigón fresco se detiene en el preciso momento en que la pasta aparece en la superficie, al no quedar huecos sin llenar, la resistencia mecánica será función principalmente de la relación agua/ /cemento de la pasta conglomerante.

En trabajos previos (9), (10), (11) y (14) se ha investigado la composición de esqueletos granulares compactos de mínima superficie específica, los cuales para una determinada cantidad de cemento y consistencia del hormigón fresco permiten reducir a un mínimo la cantidad requerida de agua de amasado. En estos trabajos se ha determinado, para cada agregado grueso componente y forma de sus partículas (angulosas o redondeadas), el módulo granulométrico del esqueleto granular compacto $(M a)$. Este, resulta ser función li- 
neal del logaritmo del correspondiente tamaño máximo nominal (Dmáx-mm), considerando como tal, al lado de la abertura de malla de un tamiz supuesto que deja pasar el $95 \%$ del total del agregado granular analizado granulométricamente.

El esqueleto granular compacto puede repoducirse, ya sea por la ley de las mezclas, partiendo de los módulos granulométricos de los agregados componentes respectivos o por composición de retenidos parciales.

En la composición de los esqueletos granulares compactos se excluyen las partículas superfinas que pasan a través del tamiz $\mathrm{n}^{\circ} 100(0,149 \mathrm{~mm})$, las que presentan marcada influencia sobre las propiedades reológicas del hormigón fresco y son consideradas como adiciones minerales activas.

El agua retenida o de mojado complementa los valores que particularizan a un cierto agregado granular, determinado por su módulo granulométrico y su tamaño máximo nominal. Permite considerar la forma de las partículas y su composición mineralógica. El agua de mojado comprende al agua adsorbida, ya que actúa por efecto de superficie quedando adherida a las partículas en la forma de capa tenue como consecuencia de la capilaridad y tensión superficial.

El agua de absorción o agua de penetración corresponde a un mecanismo diferente, ya que depende fundamentalmente de la porosidad de las partículas granulares y necesita cierto tiempo para cumplir el proceso y llegar a la saturación por inmersión en agua. De acuerdo a las experiencias de Soroker y Dovyik (15) y de Prijodko (16), sobre la absorción de los agregados granulares, se destaca la influencia que la presencia de otros componentes tiene sobre el fenómeno considerado. El poder de absorción puede reducirse en un 30 a $50 \%$, en relación a la capacidad de absorción propia de cada agregado considerado en forma aislarda.

En trabajos ya mencionados (10) y (11) se han determinado valores medios experimentales para el agua retenida o de mojado, por unidad de peso para distintos agregados normales, gruesos y finos, en relación ạl correspondiente módulo granulométrico y por extensión al esqueleto granular compacto que estos agregados componen, ya que el agua retenida total por éste último corresponde a la suma de las aguas de mojado o retenida por cada uno de los agregados intervinientes. Este agua total permite calcular la cantidad indispensable de agua de amasado para obtener una determinada consistencia del hormigón fresco, para una prefijada relación agua/cemento de la pasta conglomerante.

La consistencia se refiere al carácter de la mezcla fresca, con respecto a su estado de fluidez. Una mezcla es plástica, cuando su consistencia se encuentra entre las secas desmenuzables y las muy fluidas y es capaz de deformarse, sin segregarse. La consistencia o grado de fluidez del hormigón fresco constituye una parte importante de la trabajabilidad. Esta es una propiedad más compleja que la consistencia, puesto que involucra no solamente a las propiedades de las mezclas, sino también a las condiciones de colocación para obtener el grado requerido de consolidación, conservando la homogeneidad de cuerpo compuesto fresco. Una mezcla fresca de determinada consistencia puede ser trabajable para un determinado elemento resistente a conformar y no para otro, aparentemente muy semejante.

Para la determinación de la consistencia del hormigón fresco, uno de los procedimientos más universalmente empleados es el método del tronco de cono, de acuerdo a las Normas ASTM-C-143 e IRAM 1536, siendo la consistencia estimada en centímetros de asentamiento del tronco de cono al desmoldarse.

De acuerdo a las conclusiones de trabajos llevados a cabo hasta el presente (17), (18), (19) y (20), y de los trabajos de Papadakis (21), Soroker y Dovyik (15) y Mamillan (22), en el hormigón fresco la consistencia depende de la presencia de la pasta de cemento entre las partículas del agregado compuesto. Los esqueletos granulares compactos dan hor- 
migones cuya consistencia en estado fresco resulta ser función del espesor de la película de pasta envolvente de las partículas que lo componen. Si el esqueleto granular no es compacto y presenta gran cantidad de vacíos, parte de la pasta se pierde por ocupar estos vacíos y queda menor cantidad de pasta disponible como envolvente de las partículas componentes. De acuerdo a lo investigado al presente en nuestro Laboratorio, se puede observar que:

- la consistencia del hormigón plástico, preparado con esqueletos granulares compactos, resulta ser proporcional a la relación entre el agua de amasado y el agua de mojado o retenida total por el esqueleto granular;

- esta proporcionalidad es distinta para cada relación agua de amasado/cemento y superficie específica del cemento;

- para un mismo valor de la relación "agua de amasado/agua de mojado o retenida total", la consistencia resulta ser independiente del tamaño máximo nominal del esqueleto granular y de la composición mineralógica de los agregados normales, gruesos y finos componentes;

- para una determinada consistencia, si la pasta es de relación agua/cemento baja, hace falta mucha pasta en relación a una menor cantidad de la misma, pero de más alta relación agua/cemento.

Los álcalis del cemento influyen en la consistencia del hormigón fresco. Se disuelven rápidamente en el agua de amasado, siendo mayor su velocidad de disolución a medida que aumenta la temperatura. Generalmente, la reducción en el contenido de álcalis es acompañada por una acentuada reducción en los contenidos de ferroaluminato tetracálcico $\left(\mathrm{C}_{4} \mathrm{AF}\right)$ y de aluminato tricálcico $\left(\mathrm{C}_{3} \mathrm{~A}\right)$.

En la figura 1 se indica, para hormigones de cemento portland con agregados granulares normales y esqueletos granulares compactos, la expresión general obtenida en trabajos previos como correspondencia entre el COEFICIENTE " $\mathrm{K}$ " (relación agua de amasado/agua de mojado o retenida total), y la consistencia del hormigón fresco (Ac-cm):

$$
\mathrm{K}=a+b \cdot A c(\mathrm{~cm})
$$

válida para asentamientos entre 1,0 y $15,5 \mathrm{~cm}$ (ASTM-C-143 e IRAM 1536 ).

Los parámetros " $a$ " y " $b$ " dependen de la relación agua/cemento de la pasta conglomerante y del contenido en álcalis y superficie específica del cemento portland. Resultan ser independientes del tamaño máximo nominal, forma y composición mineralógica de las partículas naturales del esqueleto granular.

Considerando las conclusiones señaladas, en este trabajo se investiga la influencia de la finura de molido del cemento portland a través de las propiedades plásticas del microhormigón fresco, cuyo esqueleto granular es el de menor tamaño máximo nominal entre todos los esqueletos examinados.

\section{TRABAJO EXPERIMENTAL}

Se emplearon pastas conglomerantes de relaciones agua/cemento: 0,35-0,40-0,50-0,57 y 0,70 , preparadas con cemento portland de conocida composición mineralógica y finuras medias: 2.570-2.860-3.000-3.100-3.200-3.300 y $4.000 \mathrm{~cm}^{2} / \mathrm{g}$ (BLAINE). El valor indicado en primer término corresponde a la superficie específica media original, y los restantes fueron obtenidos incrementándola por molido adicional. El micro-esqueleto granular fue estructura- 
do por composición de retenidos parciales sobre tamices: $\mathrm{n}^{\circ} 30(0,59 \mathrm{~mm}), \mathrm{n} \cdot{ }^{\circ} 50(297 \mathrm{~mm})$ y n. ${ }^{\circ} 100(0,149 \mathrm{~mm})$. Las diferentes partículas tienen sus ángulos redondeados y fueron obtenidas procesando arena silícea del río Paraná, previo lavado. A este micro-esqueleto granular le corresponde:

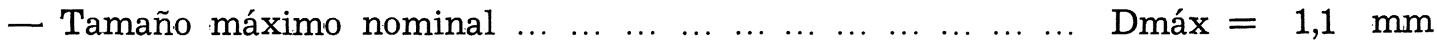

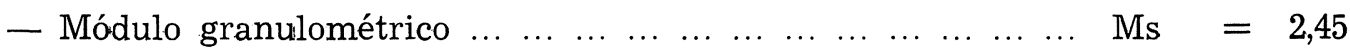

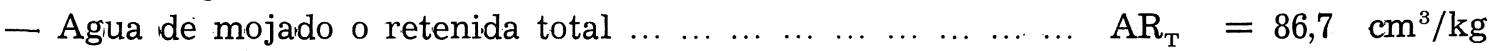

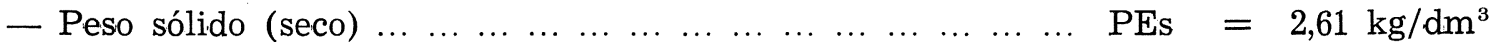

Los distintos retenidos parciales componentes del micro-esqueleto granular empleados fueron tomados en estado seco. Se mezclaron primero entre sí, y luego con el cemento portland, hasta distribución uniforme. Finalmente fue incorporada el agua de amasado, empleándose exclusivamente agua destilada. El tiempo de amasado ha sido el mismo para las diferentes preparaciones. Se seleccionaron, entre todos los pastones preparados, los de consistencia plástica con asentamientos dentro del rango: $A c=2,5 \mathrm{~cm}$ a $15,0 \mathrm{~cm}$. En cada caso, una vez concluido el amasado, se llevaron a cabo tres estimaciones de consistencia, considerándose el valor medio resultante. Las condiciones del laboratorio y de los materiales empleados fueron: $t=20 \pm 2^{\circ} \mathrm{C}$ y $\mathrm{HR}=65 \pm 5 \%$.

\section{INTERPRETACION DE LOS VALORES OBTENIDOS}

Los valores medios obtenidos se interpretan para cada relación agua/cemento adoptada, en relación con la consistencia del micro-hormigón fresco y la superficie específica del cemento empleado, para distintos valores del "COEFICIENTE K", resultando las correspondencias reflejadas en la "Tabla I".

Se observa cómo la relación agua/cemento de la pasta conglomerante y la finura de molido del cemento portland componente de la misma, gobiernan las propiedades plásticas del hormigón fresco.

Interpretando los valores de la Tabla I, se han preparado las curvas representadas en las figuras 2, 3 y 4, para asentamientos: $A c=5,0 \mathrm{~cm}, A c=10,0 \mathrm{~cm}$ y $A c=15,0 \mathrm{~cm}$. Se observa cómo puede establecerse, para una determinada consistencia, una finura óptima del cemento portland, la cual permite reducir a un mínimo el contenido necesario de agua de amasado. Para consistencias estimadas por asentamientos del tronco de cono (al desmoldarse), entre $2,5 \mathrm{~cm}$ y $15,0 \mathrm{~cm}$, la finura óptima corresponde a superficies específicas entre 2.900 y $3.060 \mathrm{~cm}^{2} / \mathrm{g}$ (BLAINE).

\section{O N C L U S I O N E S}

La relación agua/cemento de la pasta conglomerante y la finura de molido del cemento portland componente de la misma, gobiernan las propiedades del hormigón fresco.

Para una determinada consistencia puede establecerse una finura de molido óptima del cemento portland, la cual permite reducir a un mínimo los requerimientos de agua de amasado.

Estimando la finura de molido por mediciones de superficie específica puede establecerse que, para consistencias del hormigón fresco dentro del rango plástico, la superficie específica óptima corresponde a valores entre 2.900 y $3.060 \mathrm{~cm}^{2} / \mathrm{g}$ (BLAINE). 


\section{R E F E R E N C I A S}

(1) Sabesinsky Felperin, M. y OJeda, J. C.: "Influencia de la superficie específica del cemento portland, en la consistencia del hormigón fresco". XVI Jornadas Sudamericanas de Ingenieria Estructural, Bs. Aires, 1973.

(2) SAbESINSKy Felperin, M.: "Examen conductimétrico del proceso de fraguado y de endurecimiento de pastas de cemento portland y efecto de los finos de sílice, en las mismas" $\mathrm{X}$ Jornadas Sudamericanas de Ingeniería Estructural, Bs. Aires, 1965.

(3) SAbesinsky Felperin, M.: "Conductimetric examination of the setting and hardening process of airentrained micro-concrete, for different consistency and water/cement ratios". RILEM Simposium on admixtures, Bruselas, 1967.

(4) SABesinski FELPERIN, M.: "El mortero RILEM/CEMBUREAU. Influencia de los finos de silice a través del examen conductimétrico del proceso de fraguado". Grupo latinoamericano de la RILEM, INTI, Bs. Aires, 1968

(5) SAbesinsky Felperin, M.: "Influencia del esqueleto granular en el mortero RILEM/CEMBUREAU, por efecto de los finos de sílice. 2. ${ }^{\text {a }}$ parte". Simposio sobre aglomerantes hidráulicos y sus aplicaciones, LEMIT, La Plata, 1970.

(6) SAbesinsky Felperin, M.: "Conductimetric study of the influence of retarding admixtures on the setting process in RILEM/CEMBUREAU mortar under high- temperature conditions". International RILEM Simposium on concrete and reinforced concrete in hot countries, Haifa, 1971.

(7) Rebinder, P. A.: "La mecánica físico-química” Academia de Ciencias, Moscú, 1958.

(8) JASPERS, M. J. M.: "Relation between specific surface and other properties of cement". Cement and Lime Manufacture. Vol. XXVI, n. 1, 1953.

(9) SAbesinski Felperin, M.: “Método para proyecto de hormigones". I Simposio sobre materiales. LEMIT, La Plata, 1964

(10) Sabesinsky Felperin, M.: "Composición de agregados compactos, gruesos y compuestos, para proyecto de hormigones normales de distintas consistencias y relaciones agua/cemento". XIII Jornadas Sudaméricanas de Ingeniería Estructural, Montevideo, 1969.

(11) Sabesinsky Felperin, M.: "Influencia del tamaño máximo del esqueleto granular, en el proyecto de hormigones de cemento portland". Reunión conjunta de las XIV Jornadas Sudamericanas de Ingeniería Estructural y el IV Simposio Panamericano de Estructuras, Bs. Aires, 1970.

(12) SABesinsky Felperin, M.: "Micro-hormigones de cemento portland. Influencia de la pasta conglomerante en la incorporación intencional de aire". IV Simposio sobre aglomerantes hidráulicos, hormigones, áridos y sus aplicaciones, LEMIT, La Plata, 1972

(13) RichaRtz, W.: "Acerca del desarrollo de la estructura y la resistencia de la pasta de cemento". Materiales de Construcción, n. ${ }^{\circ}$ 140, Instituto Eduardo Torroja, Madrid, 1970.

(14) Sabesinsky Felperin, M.: "Hormigones de cemento portland con agregados normales. Relación entre el tamaño máximo del esqueleto granular y el agua de amasado, para distintas consistencias y relaciones agua/cemento". Materiales de Construcción, n. 143, Instituto E. Torroja, Madrid, 1971.

(15) Soroker, V. I. y Dovyik, V. G.: "Consistentes mezclas de hormigón. Elaboración de elementos premoldeados". Edit. Literatura de la Construcción (en ruso), Moscú, 1964.

(16) Prijodko, O. M.: "Dependencia de la resistencia al frío del hormigón de kerancita, de la estructura y de la porosidad". Publicación (en ruso), Hormigón y Hormigón Armado, n. ${ }^{\circ}$ 10, Moscú, 1967.

(17) SABesinsky FeLPeRIN, M.: "El agua de amasado en la consistencia del hormigón fresco, para distintas relaciones agua/cemento". IMAE, 1966.

18) SAbesinski Felperin, M.: "Interaction in fresh micro-concrete, between air-entrained and consistency, for different water/cement ratios". International RILEM Symposium on Admixtures for Mortar and Concrete, Bruselas, 1967.

(19) SAbesinsky FELPERIN, M.: "The portland cement paste, in the shrinkage of micro-concretes, for different water/cement ratios". International RILEM/CEMBUREAU Symposium on Shrinkage on Hydraulic Concrete. Madrid, 1968.

(20) Sabesinsky Felperin, M.: "Contenido óptimo de aire en los hormigones de cemento portland". XV Jornadas Sudamericanas de Ingeniería Estructural, Porto Alegre, 1971.

(21) Papadakis, M.: "Rôle des éléments fins dans la Rhéologie du Béton Frais". París, 1963.

(22) Mamillan, M.: "Les propriétés mécaniques du Béton frais pour la préfabrication". Annales Bâtiment et Travaux Publics, n.o 201, Set., 1964 
T A B L A I

Valores del coeficiente $K\left(K=A a / A R_{\mathrm{T}}\right)$, correspondientes a consistencias del hormigón fresco, dentro del rango plástico, en función de la finura de molido del cemento portland (superficie específica - $\left.\mathrm{cm}^{2} / g-B L A I N E\right)$, y de la relación agua/cemento $(A a / C)$, de la pasta conglomerante.

Ac (cm)-consistencia estimada por asentamientos s/ASTM-C-143 e.IRAM 1.536

\begin{tabular}{|c|c|c|c|c|c|c|c|c|}
\hline \multirow[b]{2}{*}{$\mathrm{Aa} / \mathrm{C}$} & \multirow[b]{2}{*}{$\begin{array}{r}\mathrm{Ac} \\
(\mathrm{cm})\end{array}$} & \multicolumn{7}{|c|}{ SUPERFICIE ESPECIFICA DEL CEMENTO PORTLAND } \\
\hline & & 2570 & 2,850 & 3000 & 3100 & 3200 & 3300 & 4400 \\
\hline \multirow{6}{*}{0,35} & 2,5 & 2,40 & 2,20 & 2,11 & 2,16 & 2,13 & 2,17 & 2,33 \\
\hline & 5,0 & 2,53 & 2,41 & 2,32 & 2,35 & 2,36 & 2,39 & 2,67 \\
\hline & 7,5 & 2,65 & 2,58 & $2, \underline{52}$ & 2,57 & 2,58 & 2,60 & 3,03 \\
\hline & 10,0 & 2,77 & 3,04 & 2,72 & 2,77 & 2,82 & 2,81 & 3,37 \\
\hline & 12,5 & 2,91 & 2,98 & 2,93 & 2,98 & 3,03 & 3,05 & 3,75 \\
\hline & 15,0 & 3,02 & 3,17 & 3,14 & 3,19 & 3,26 & 3,27 & 4,04 \\
\hline \multirow{6}{*}{0,40} & 2,5 & 2,25 & 2,01 & 1,95 & 1,96 & 1,96 & 1,98 & 2.05 \\
\hline & 5,0 & 2,31 & 2,11 & 2,07 & 2,08 & 2,10 & 2,14 & 2,29 \\
\hline & 7,5 & 2.38 & 2,19 & 2.17 & 2.20 & 2,23 & 2,29 & 2,50 \\
\hline & 10,0 & 2,44 & 2,28 & 2,28 & 2,30 & 2,37 & 2,42 & 2,70 \\
\hline & 12,5 & 2,51 & 2,37 & 2,40 & 2,42 & 2,49 & 2,57 & 2,90 \\
\hline & 15,0 & 2,57 & 2,47 & 2,52 & 2,54 & 2,63 & 2,72 & 3,13 \\
\hline \multirow{6}{*}{0,50} & 2.5 & 2.14 & 1,93 & 1.86 & 1.85 & 1.87 & 1,86 & 1.76 \\
\hline & 5,0 & 2,18 & 1,99 & 1,93 & 1,93 & 1,98 & 1,99 & 1,87 \\
\hline & 7,5 & 2,21 & 2,05 & 2,00 & 2,00 & 2,07 & 2,09 & 1,98 \\
\hline & 10,0 & 2,25 & 2,10 & 2,07 & 2,08 & 2,17 & 2,20 & 2,09 \\
\hline & 12,5 & 2,28 & 2,15 & 2,14 & 2,15 & 2,25 & 2,31 & 2,19 \\
\hline & 15,0 & 2,31 & 2,22 & 2,21 & 2,23 & 2,35 & 2,42 & 2,30 \\
\hline \multirow{6}{*}{0,57} & 2,5 & 2,17 & 1,94 & 1,88 & 1,89 & 1,89 & 1,91 & 1,81 \\
\hline & 5,0 & 2,21 & 2,01 & 1,96 & 1,97 & 2,00 & 2,02 & 1,92 \\
\hline & 7.5 & 2,24 & 2.06 & 2.04 & 2.05 & 2.11 & 2.14 & 2.03 \\
\hline & 10,0 & 2,29 & 2,13 & 2,13 & 2,14 & 2,22 & 2,25 & 2,14 \\
\hline & 12.5 & 2,32 & 2,20 & 2,21 & 2,22 & 2,32 & 2,35 & 2,25 \\
\hline & 15,0 & 2,35 & 2,26 & 2,30 & 2,31 & 2,43 & 2,47 & 2,36 \\
\hline \multirow{6}{*}{0,70} & 2,5 & 2,19 & 1,95 & 1,92 & 1,96 & 1,92 & 1,91 & 1,92 \\
\hline & 5,0 & 2,23 & 2,74 & 2,01 & 2,04 & 2,03 & 2,03 & 2,05 \\
\hline & 7.5 & 2.27 & 2,10 & 2.10 & 2.11 & 2,12 & 2.14 & 2,16 \\
\hline & 10,0 & 2,31 & 2,19 & 2,19 & 2,20 & 2,24 & 2,26 & 2,29 \\
\hline & 12,5 & 2,35 & 2,26 & 2,27 & 2,27 & 2,34 & 2,39 & 2,42 \\
\hline & 15,0 & 2,40 & $2 ; 34$ & 2,36 & 2,35 & 2,44 & 2,51 & 2,53 \\
\hline
\end{tabular}



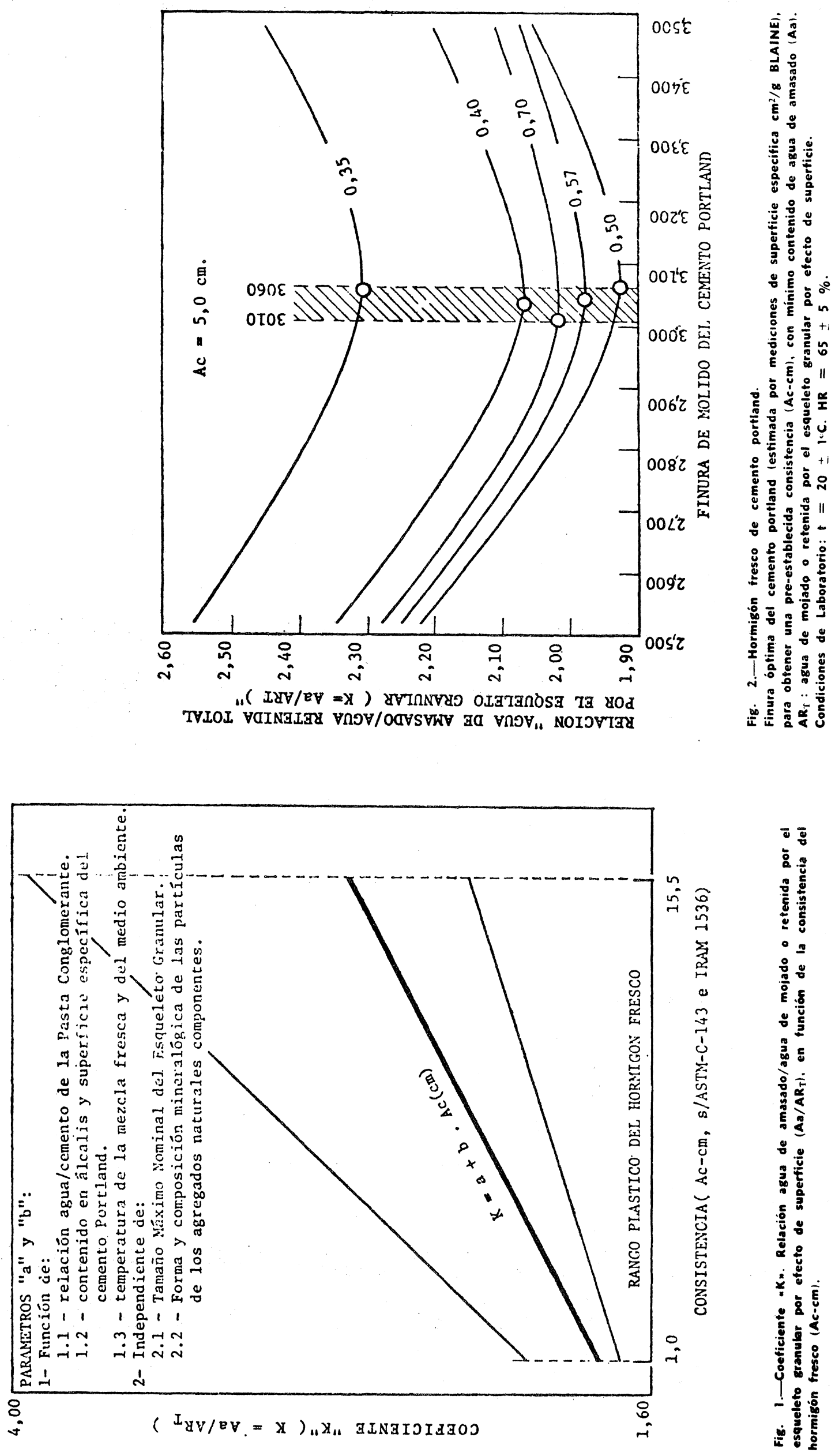

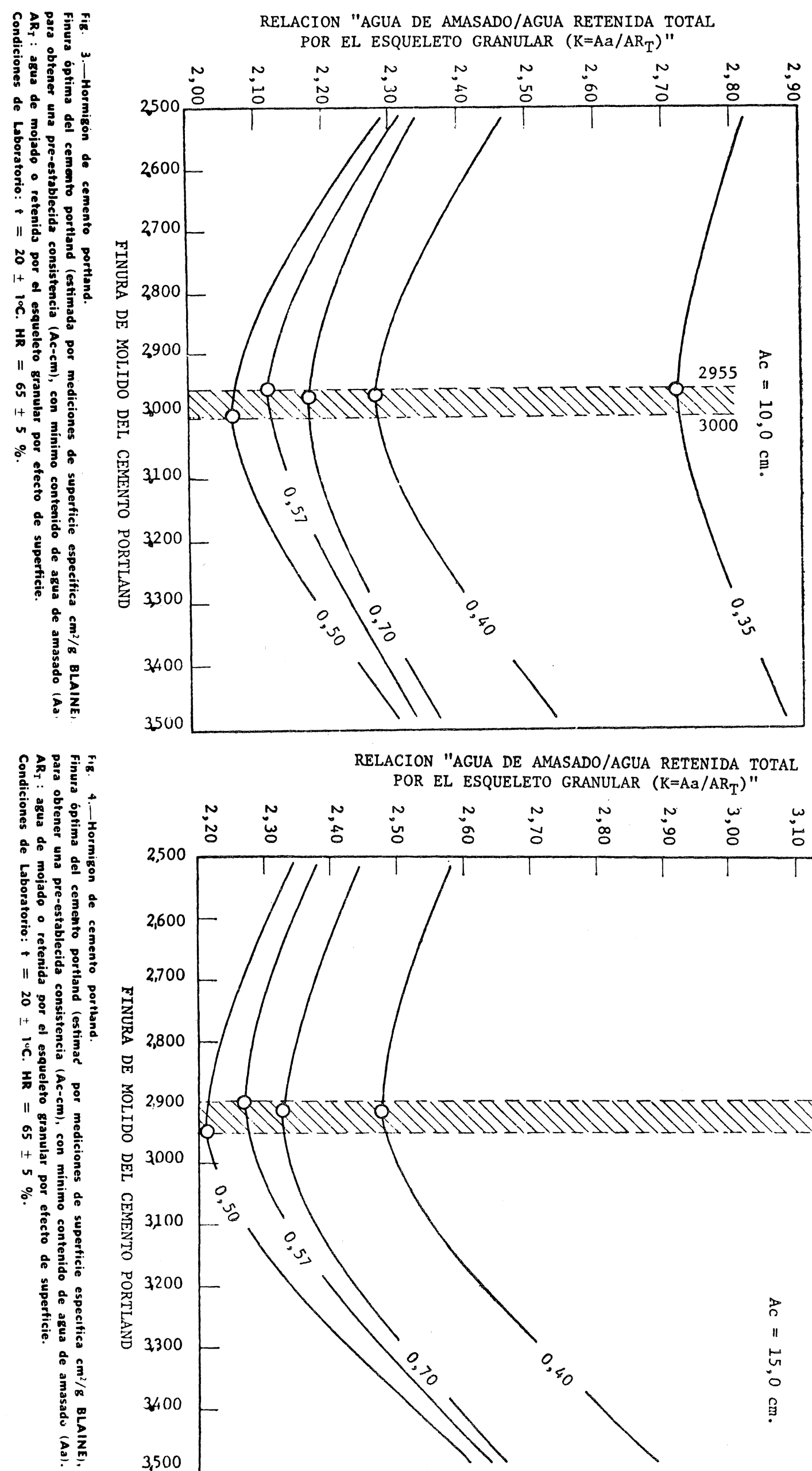

RELACION "AGUA DE AMASADO/AGUA RETENIDA TOTAL POR EL ESQUELETO GRANULAR $\left(\mathrm{K}=\mathrm{Aa} / \mathrm{AR}_{\mathrm{T}}\right)$ "

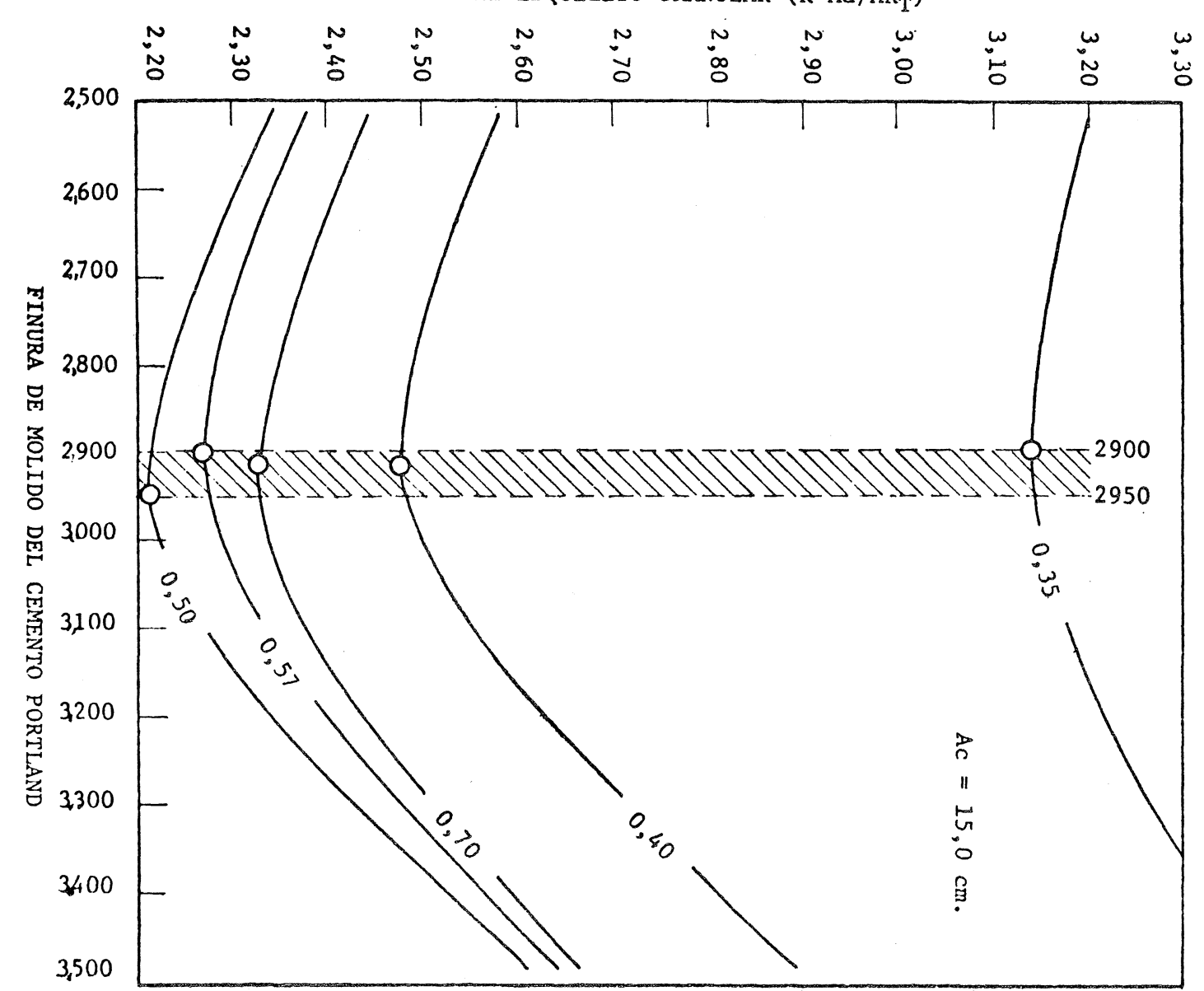

